\title{
Independent Zone Setup Scheme for Re-configurable Wireless Network
}

\author{
Jae-Pil Yoo, ${ }^{1}$ Kee-cheon Kim, and SunYoung Han ${ }^{1}$ \\ School of Computer Science \& Engineering. Konkuk University, Seoul, Korea, \\ $+82-2-450-3518$
}

\begin{abstract}
ZRP (Zone Routing Protocol), is especially considered to be suitable for moves of dynamic nodes and scalability of RWN. In configuring the ZRP zone, if we remove redundant function of a node, we can get performance improvement in routing. This improved performance of routing is usually from configuring a ZRP zone in which a node doest not have functioning zone head, relaying (internal) node, and peripheral node simultaneously. In such cases, internal routing information size based on link state can be significantly reduced. Hop count is also reduced when routing is reduced and routing loop prevention steps are taken. In this paper $^{1}$ we introduce a zone setup scheme based on ZRP in which a node does not have overlapping function as head and relay, and border. Also we introduce a routing scheme for proposed new zone setup scheme.
\end{abstract}

\section{Introduction}

RWN (Re-configurable Wireless network) is a scalable network in which a number of nodes can moves around dynamically. A node itself has a sending or receiving wireless interface and with these interfaces nodes can communication each other. When they communicate each other, each node needs to relay some traffic for others without relying on fixed transmission infrastructure. We assume that almost all the nodes in RWN have limited wireless transmission radius and power supply.

Routing protocol that reflects features mentioned above is classified into two types: proactive scheme and reactive scheme. Proactive is a scheme that all the nodes constantly and periodically exchange network path information prior to sending packets such as OSPF [1]. On the other hand, reactive scheme sends path setup signal only when it is needed. It is also called 'on-demand' scheme $[2][3]$. But it needs packet buffering. Since all the nodes using proactive scheme already know their destination path information before they send data traffic, they are able to send data traffic without delay or buffering. In spite of its merits, the use of proactive scheme heavily depends on the number of nodes in RWN. Therefore, it has a scalability problem. In order to overcome this problem, various clustering (grouping) scheme were introduced [4][5][6][7][8]. Clustering scheme is

1 This work was supported by grant No. R01-2001-000-00349-0(2002) from the Basic

Re-search Program of the Korea Science Engineering Foundation. 
to grouping local nodes into a cluster and then separates inter-cluster routing information and intra-cluster routing information for routing efficiency. One of above scheme is ZRP, ZRP uses hybrid scheme; proactive method for intra-zone routing such as link-state and on-demand based reactive method for inter-zone routing for dynamic RWN

\section{ZRP and Independent Zone Setup Scheme for RWN}

$\mathrm{ZRP}$ is a hybrid of reactive and proactive scheme [9][10]. A zone is established as setting the zone center node as the central point of zone and the zone center node has n-hop radius within the zone, in which nodes make maximum n-hop path. Intra-zone routing protocol such as IARP monitors intra-zone routing information and keeps routing table constantly. Inter-zone routing protocol such as IERP finds paths using so-called border-casting. If the zone center node named zone head needs to send some packets to the destination, it sends query packets to its peripheral nodes using border-casting, which groups the same zone. At the same time, one of peripheral nodes that receives query packet also functions as a center node of a certain zone. This node checks whether the destination node resides in its zone or not. If the destination exists, that node sends reply packet to the source node in the reverse order. If not, it repeats border-cast to its peripheral nodes. When a query packet travels around zone's center nodes and peripheral nodes, it adds node's unique ID to its packet header. Finally, when a source node receives a reply packet, it sets reverse path of the reply packet as a routing path. In case of ZRP routing, all the nodes have their zones and also belong to other zones. Each node has functions of zone head, relaying (internal) nodes, and peripheral nodes simultaneously. The number of zones matches exactly with the number of nodes. It means that the number of different intra-zone routing information is set on each zone. Figure 1 Depicts ZRP zone topology. Solid line circles (cells) are needed to route from the source 'node S' to the destination 'node D'. Dotted line circles (cells) also represent each zone, but it does not participate in routing from 'node $\mathrm{S}$ ' to 'node $\mathrm{D}$ '

However, if we possibly prevent a zone area from overlapping each other, and we only permit overlapping peripheral nodes, each node in RWN should function as a center node, internal node, or peripheral node. A node will function as a center node, peripheral node or a relay node when setting up a zone. This removal of overlapping zones results in simplification of node function and routing efficiency and it also reduce the traffic that is used to prevent the routing loop. In case of the existing ZRP, each node belongs to many different zones depending on n-hop radius. It means that if a node belongs to ten different zones, it must monitor different ten routing information. But if we keep zones not to be overlapped, a node does not have to function as a center, internal, and peripheral node at the same time, all the nodes in a zone only need to keep its internal routing information.

At this time, the only problem is to configure the initial zone that does not overlap and keeps the zone without overlaps constantly. If we just adopt 


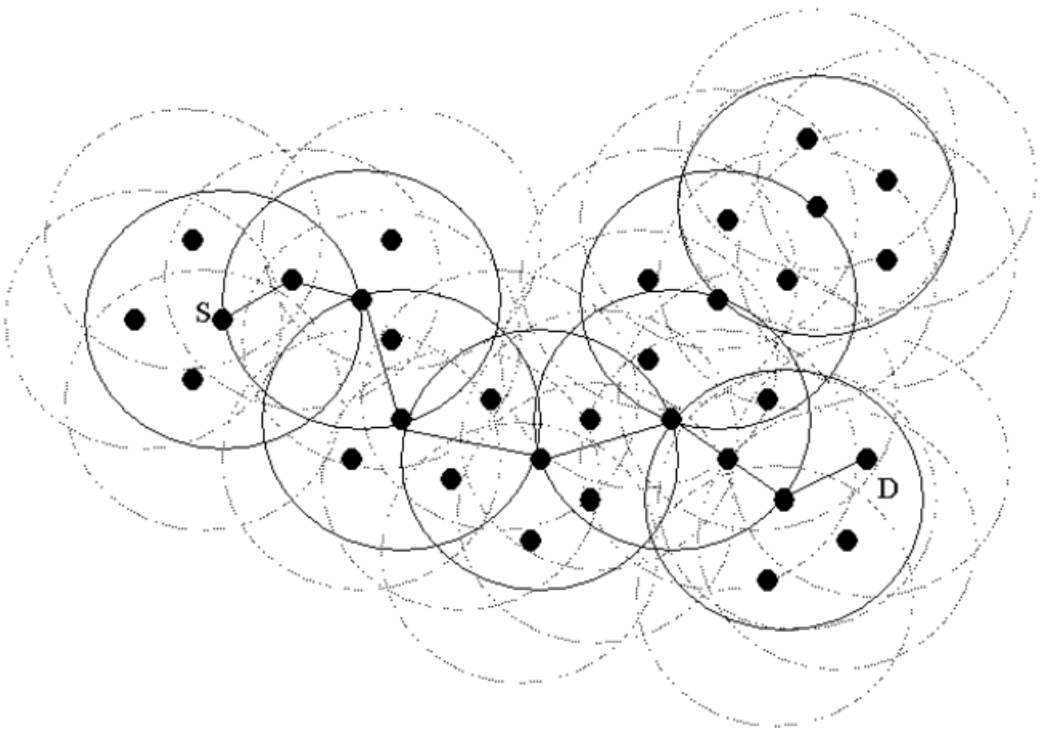

Fig. 1. Zone Topology of ZRP and an example of its IERP operation

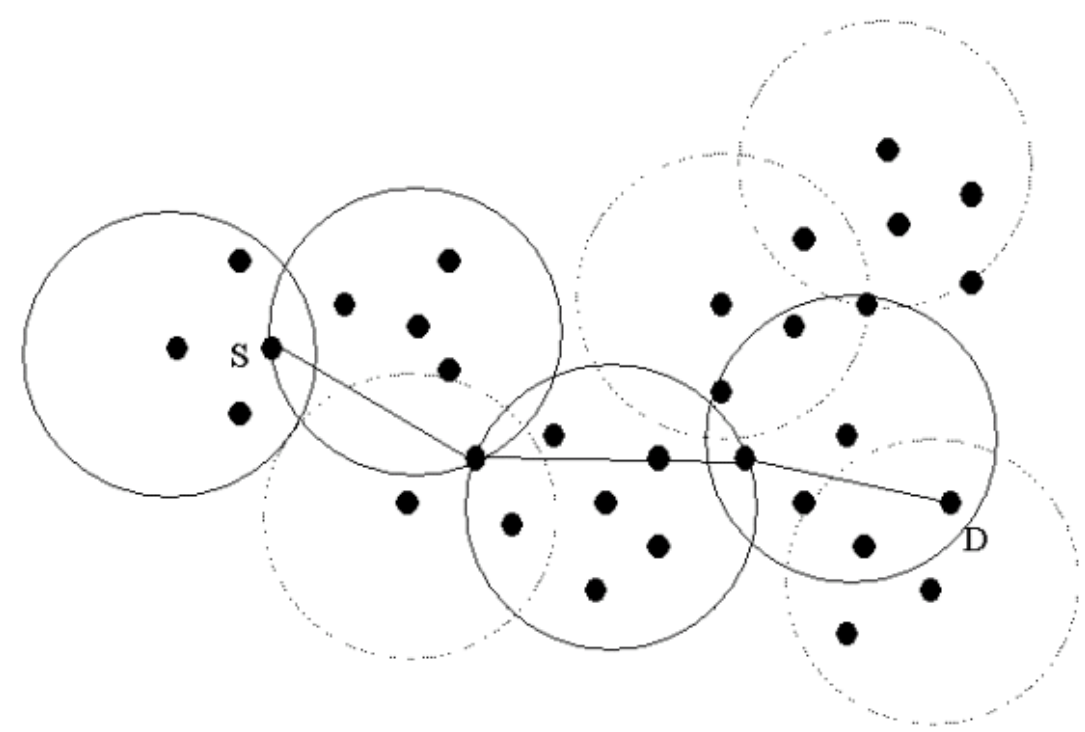

Fig. 2. Newly configured Independent Zone Topology and an example of its IERP operation 
previous research result for setting up a simple cluster or hierarchical cluster [5][6], it is not easy to reflect zone radius of ZRP. It results in imbalance of each zone. After all, we need new algorithm for a zone configuration that does not overlap that named independent zone. We also need a scheme that maintains overlap-free zones even when the node moves and zones with modified routing schemes. Figure 2 depicts a newly configured independent zone topology and an example of its IERP operation. Solid line circles (cells) are needed to route from the source 'node S' to the destination 'node D'. Dotted line circles (cells) also mean each zone, but it does not participate in routing from 'node $\mathrm{S}$ ' to 'node D'. The details of its operation will be explained in the coming sections of this paper.

\section{Algorithms for Independent Zone Scheme}

\subsection{Independent Zone Configuration}

Algorithm as follows, tries to make a zone without overlaps during the initial zone configuration allowing as many nodes as possible in a zone radius. Once a zone is configured, it starts making another zone and shares a peripheral node that belongs to each zone to make a connection from one to others.

a) Each node finds out the number of the neighbor node contained within suitable signal strength. Such signal strength also becomes an n-hop radius of independent zone.

b) Each node exchanges neighbor counts with its neighbors. A node that has maximum neighbor node count becomes the center node of an independent zone. It informs their neighbors that it is the center node of the zone for them to join a specific zone. It implicitly tells that all the nodes once joined to a specific zone are not to respond to a zone configuration request except for the peripheral nodes. (Center node for zone X: cX, internal node for zone $\mathrm{X}$ : iX, peripheral node for zone X: $\mathrm{pX}$ )

c) This step finds candidate zones. A center node for zone X, cX, selects one of its peripheral nodes, $\mathrm{pX}$, and delegates $\mathrm{pX}$ to configure the zone by sending request message. $\mathrm{pX}$ temporarily becomes a center node, $\mathrm{cA}$, of the virtual zone A. And then, $\mathrm{cA}(=\mathrm{pX})$ tries to find out the peripheral node p'A that has the largest neighbor count in its 'virtual zone A'. Undoubtedly, neighbor count means the number of nodes that does not configure the zone at that time. To find out a node that has the largest number of neighbors, we need to just apply step a). If the nodes are uniformly distributed, the peripheral node p'A with the largest neighbor count would be located in a straight line from cX via pX, that is to say, p'A will be the farthest peripheral node belongs to the virtual zone A from cX. This scheme makes each zone not to be overlapped each other and allows a zone to have the maximum number of nodes as possible. Fig.3 depicts this step.

d) p'A with the largest neighbor count configures a new zone Y. At the same time, p'A becomes a center node cY of zone Y. Step b) can be applied in 
configuring the zone. At this time, pX naturally belong to the zone Y. so pX also becomes pXY, it then acts as a peripheral node of zone $\mathrm{X}$ and zone $\mathrm{Y}$ at the same time.

e) Repeat step c) and d) to extend the zone coverage. If a node heard requesting messages to configure a zone that already belongs to a certain zone, it just discards the requesting message.

f) During the step 1 through 5 , if some nodes could not configure a zone for the critical time value, they just make zones with them. Also, step b) can be applied in configuring the zones. However, a configured zone needs to have at least on minimum peripheral node sharing with other zones to constitute a routing path.

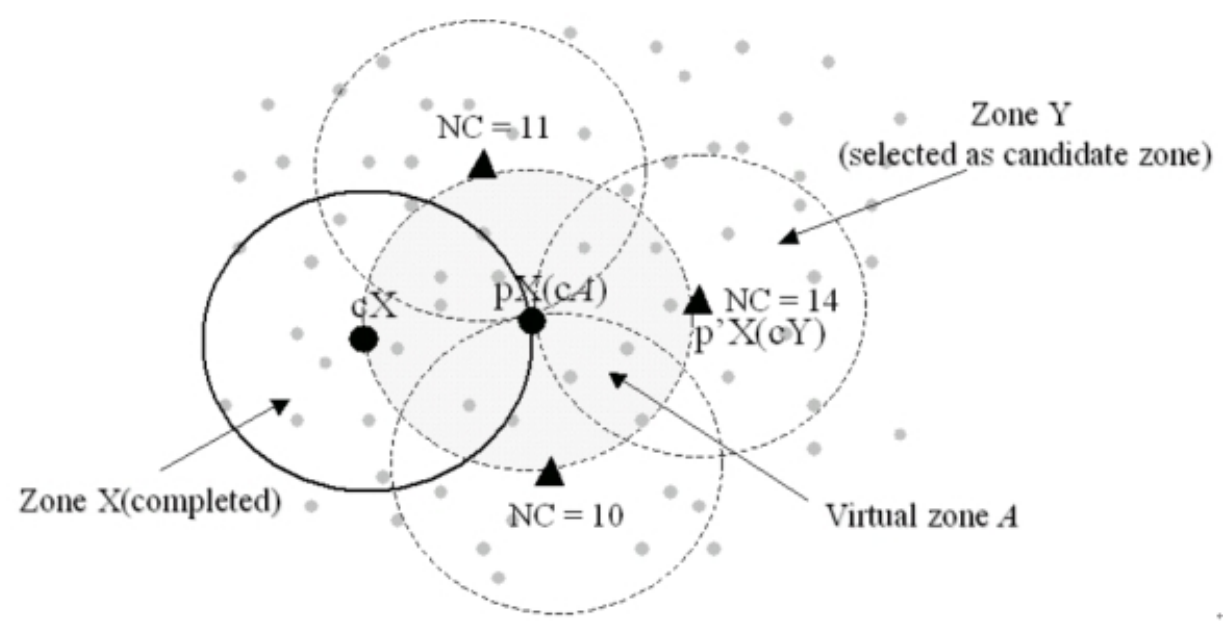

Fig. 3. Finding next candidate zone

\subsection{Independent Zone Maintenance}

a) Movement of a node within a zone does not affect the external routings. It only affects the internal routing information. So it easily fixes up the internal routing information changes

b) If a peripheral node of a zone moves to another location, and if the peripheral node just breaks routing path between two zones, one of the center node of two zones needs to strengthen the signal power to find out the substitute peripheral node to connect the two broken zones.

c) If the center node of a zone moves to another location, and if the nodes within the zone cannot communicate each other, the zone should be divided into two zones to restore the connection. Some time later, if some nodes reach a location that connects the split zones into one, they can be merged. 


\subsection{Independent Zone Routing}

A source node s of a zone $\mathrm{X}, \mathrm{sX}$, needs to follow the following steps to find a routable path to the destination node $\mathrm{d}$ of zone $\mathrm{Z}, \mathrm{dZ}$.

a) $\mathrm{sX}$ tries to find out $\mathrm{dZ}$ in its routing table belongs to zone $\mathrm{X}$. if $\mathrm{dZ}$ is found in the internal routing table of $\mathrm{sX}, \mathrm{sX}$ can send packets to $\mathrm{dZ}$ directly .

b) If sX could not find $\mathrm{dZ}, \mathrm{sX}$ border-casts query message to all the peripheral nodes $\mathrm{pX}$ in the zone $\mathrm{X}$.

c) One of the peripheral node $\mathrm{pX}$ in zone $\mathrm{X}$ may also belongs to other zones and also has many routing tables depends on the number of zones it belongs to. So, pX that receives a query message tries to find out the destination node $\mathrm{dZ}$ in its routing table except for the zone from which query message arrived.

d) If $\mathrm{dZ}$ is not found in each routing table on the peripheral node, $\mathrm{pX}$ bordercasts the query message to the rest of the peripheral nodes except for the zone from which query message came.

e) Repeats c), d) until source node sX finds the destination node dZ.

\section{Performance Evaluation}

The results of our simulation are presented in the following graphs. We use the assumption that a network of 200 nodes moves in an area of $1000 \mathrm{x} 1000 \mathrm{~m}$ Squares. In this model every node can select a random location and moves there at a uniformly chosen speed of between 0 and $1 \mathrm{~m} / \mathrm{s}$. We ignored bordercasting delay and IARP retrieving delay setting as $0 \mathrm{~ms}$ respectively. A node can send query packets by maximum of ten times in one second.

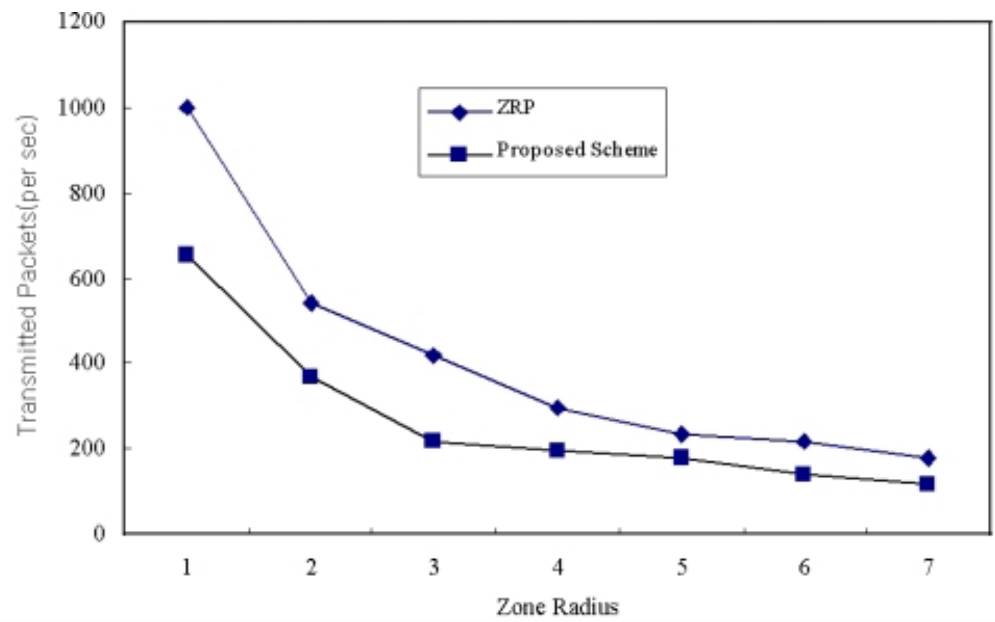

Fig. 4. Traffic per Route Query after initial setup 
Figure 4 illustrates the transmitted packets of basic ZRP and the proposed scheme supporting the independent zone with IERP operation. The number of transmitted packets depends on the zone radius. But, in most cases, the proposed scheme shows less IERP operation packets in a network. IERP Operation is mostly depends on the number of zones. So it is natural that the proposed scheme is more efficient than the basic ZRP, because the proposed scheme has by far less number of zones than that of the basic ZRP. Besides, the proposed scheme just needs nearly half the number of zones than the basic ZRP with IERP operation.

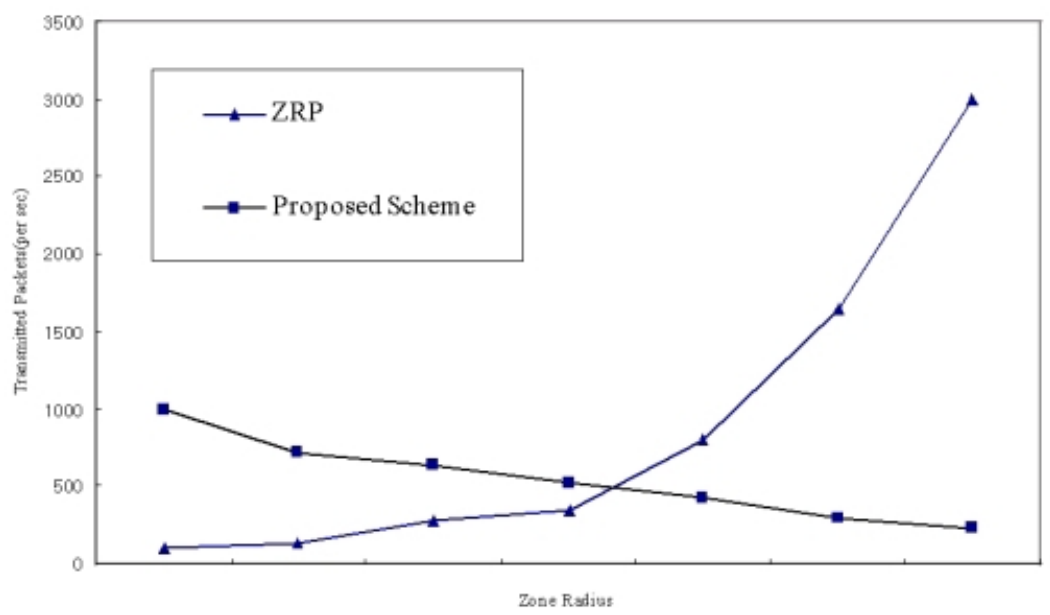

Fig. 5. Control Traffic variation due to mobile

Figure 5 illustrates the transmitted packets of basic ZRP and the proposed scheme supporting the independent zone with re-configuration due to the movement of a node. Basic ZRP shows when a zone radius grows larger, much more control traffic flows on the network. In case of the proposed scheme, when its zone radius is small, it uses much traffic to re-configure the zone than the basic ZRP. However, as the zone radius grows larger, it uses less traffic to re-configure the zone in comparison with the basic ZRP. We expect that intra-zone movement and inter-zone movement ratio causes this result. With the smaller zone radius, almost all the movement of node are considered to be the inter-zone movement. So it needs to re-configure the zone frequently. But the larger zone radius means more inter-zone movement. So it will have less chance to re-configure the zones.

\section{Conclusion}

In this paper, we proposed a new independent zone configuration scheme for ZRP in which a node does not have multiple functions as head, internal, 
and peripheral node. We also introduced a modified routing scheme for the newly established zone scheme. This scheme tries to make a zone without overlaps during the initial zone configuration and allows as many nodes as possible in a zone radius. This new independent zone configuration scheme has the following advantages as shown in the performance evaluation. Internal routing information size based on the link state can be significantly reduced. A hop count is reduced and the routing loop prevention steps are possible with less cost. Out simulation results tells us that our independent zone scheme is more suitable for less dynamic large RWN.

Zone re-configuration algorithm of the proposed scheme is more complex than ZRP since It needs to keep the zone not to be overlapped all the time. This produces negative performance especially for dynamically moving node in narrow radius zone. Since the performance of RWN is affected by such parameters as node movement and zone radius, as a future research, we will research on the dynamically adaptive RWN to such parameters to enhance the performance.

\section{References}

1. J. Moy., OSPF version 2. RFC 2178, Internet Engineering Task Force, July 1997.

2. C.E. Perkins and E.M. Royer., Ad-Hoc On-Demand Distance Vector Routing. In Proceedings of the Second Annual IEEE Workshop on Wireless and Mobile Computing Systems and Applications, February 1999.

3. J.J.Garcia-Luna-Aceves and M.Spohn, Source-Tree Routing in Wireless Networks, In Proc, IEEE ICNP 99, th Intl, Conf. On Network Protocols, Toronto, Canada, Oct 1999.

4. D.J. Baker and A. Ephremides., A Distributed Algorithm for Organizing Mobile Radio Telecommunication Networks. In Proceedings of the Second International Conference on Distributed Computer Systems, April 1981.

5. C.-C. Chiang., Routing in Clustered Multi-hop, Mobile Wireless Networks.In Proceeding of ICOIN Nobemver, 1996.

6. M. Gerla and J.T.-C. Tsai, Multi-cluster, Mobile, Multimedia Radio Network, Wireless Networks, October 1995.

7. R. Krishnan, R. Ramanathan, and M. Steenstrup., Optimization Algorithms for Large Self-Structuring Networks. In Proceedings of IEEE INFOCOM '99, March 1999.

8. J. Zavgren., NTDR Mobility Management Protocols and Procedures. In Proceedings of the IEEE Military Communications Conference, November 1997.

9. Z.J. Hass and M.R. Pearlman., The Performance of Query Control Schemes for the Zone Routing Protocol. In Proceedings of SIGCOMM '98, September 1998.

10. M.R. Pearlman and Z.J. Haas., Determining the Optimal Configuration of the Zone Routing Protocol. IEEE Journal on Selected Areas of Communications, August 1999. 